\title{
Deubiquitinating ALDH1A3 key to maintaining the culprit of aggressive brain cancer
}

\author{
Hiroaki Wakimoto \\ Department of Neurosurgery, Massachusetts General Hospital, Harvard Medical School, Boston, Massachusetts, USA.
}

\begin{abstract}
Cancer stem cells sustain propagation of the deadly primary brain cancer glioblastoma. Clioblastoma stem cells (CSCs) characterized by a mesenchymal phenotype are aggressive and resistant to therapies and represent a crucial therapeutic target. In this issue of the JCI, Chen et al. show that the intracellular levels of aldehyde dehydrogenase $1 \mathrm{A3}$ (ALDH1A3), known as a functional marker of mesenchymal CSCs, are regulated posttranslationally by ubiquitin-specific protease $9 X$-mediated (USP9X-mediated) deubiquitination. Increased expression of USP9X stabilizes ALDH1A3, enabling GSCs to exhibit mesenchymal traits and the malignant phenotype. Thus, the USP9X-ALDH1A3 axis may offer a novel therapeutic target in glioblastoma.
\end{abstract}

\section{Mesenchymal glioblastoma stem cells}

Despite much-improved understanding of underlying molecular pathogenesis, glioblastoma remains one of the deadliest solid cancers in the body. In spite of the latest multimodality treatment, the median survival still remains at only 15 to 18 months (1). Since the discovery of small subsets of neoplastic cells in glioblastoma with stem-like properties and high tumorigenicity, glioblastoma stem cells (GSCs), often marked by CD133 or Sox2, have constantly been a subject of extensive laboratory and clinical research. GSCs sit at the top of the hierarchy of functionally diverse tumor cells, and drive and sustain tumor propagation (2). While whether GSCs are generally more resistant to conventional therapies than the bulk tumor cells is controversial, there is a consensus that GSCs that survive radiation therapy and chemotherapy ultimately cause a relapse of glioblastoma for which standard or effective therapy does not exist. Being genetically and phenotypically highly heterogeneous between patients, glioblastoma has been widely classified according to transcriptomebased gene expression signatures into proneural, neural, classical, and mesenchymal glioblastoma (3). Reflecting this heterogeneity, GSCs exhibit contrasting expression profiles, either proneural or mesenchymal (4). In 2013, Mao et al. showed that mesenchymal GSCs were more aggressive in vivo and refractory to radiation therapy (4), identifying characteristic GSC populations that may be enriched in recurrent glioblastoma through a phenomenon analogous to the "epithelial-to-mesenchymal transition." In line with the prior report that ALDH1 facilitated GSCs (5), high activity of aldehyde dehydrogenase 1A3 (ALDH1A3) drove active glycolytic metabolism that was critical for the maintenance of mesenchymal GSCs (4). A member of the ALDH superfamily, ALDH1A3, along with ALDH1A1, ALDH1A2, and ALDH8A1, participates in the $\mathrm{NAD}^{+}$dependent oxidization of retinaldehyde to biosynthesize retinoic acid (RA) and plays a role in a number of metabolic and physiolog-

Delated Article: p. 2043

Conflict of interest: HW has an institutional sponsored research agreement with Forma Therapeutics.

ical processes (6). What is unique about ALDH1A3 is that it serves as a functional marker of mesenchymal GSCs, as well as other cancer stem cells, because its actions directly contribute to the properties of cancer stem cells and allow flow cytometry-based identification of cells with high ALDH activity, i.e., ALDH ${ }^{\text {bright }}$ cells, using the ALDEFLUOR assay. Therefore, ALDH1A3 represents a prime therapeutic target in mesenchymal GSCs. Cheng et al. modified a natural ALDH inhibitor, daidzin, and developed a compound, GA11, that upon systemic administration demonstrated in vivo efficacy in mice bearing GSC-derived xenografts (7). Regulators that control ALDH1A3 expression include FOXD1, STAT3, K-ras, HGF/c-Met, and miR-187/miR-125a/b (6), but whether targeting any of these would affect the mesenchymal GSCs through inhibition of ALDH1A3 remains unknown. Given the dearth of approaches to inhibiting ALDH1A3, a deeper understanding of how the protein levels of ALDH1A3 are regulated in mesenchymal GSCs is necessary for therapeutically targeting this critical metabolic enzyme and pathway.

\section{Role of USP9X in human mesenchymal CSCs}

Chen and colleagues discovered a new mechanism that controls the stability of ALDH1A3 in human glioblastoma cells (8). Through an siRNA screen of 98 deubiquitylating/deubiquitinating enzymes (DUBs), they identified ubiquitin-specific protease 9X (USP9X) as a specific regulator of ALDH1A3 in human mesenchymal GSCs. Upon interaction with ALDH1A3, USP9X removes Lys48-linked polyubiquitin chains of ALDH1A3, and increases ALDH1A3 stability by averting its targeting to proteasomal degradation. USP9X ${ }^{\text {high }}$ GSCs have higher ALDH activity and a higher capability to generate orthotopic gliomas in mice, as compared with USP9X ${ }^{\text {low }}$ GSCs. Genetic knockdown of USP9X reduces expression of ALDH1A3 as well as a number of other 
A

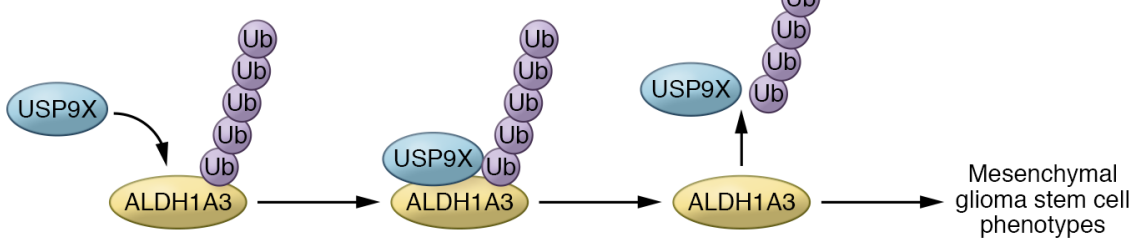

B

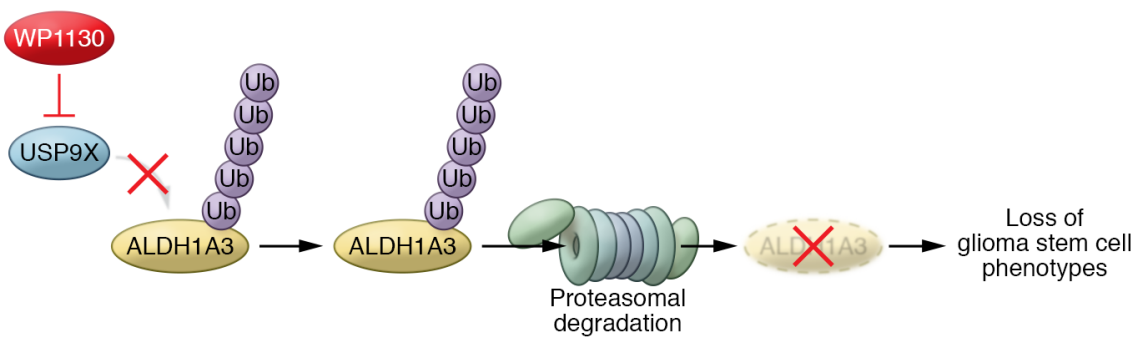

Figure 1. USP9X stabilizes ALDH1A3 to maintain mesenchymal glioma stem cells. (A) In mesenchymal glioma stem cells, Lys48-linked polyubiquitination of ALDH1A3 is rapidly targeted by the deubiquitinating enzyme USP9X, resulting in increased stability and high-level expression of ALDH1A3 that facilitates cancer stem cell phenotypes. (B) Deubiquitinating enzyme inhibitor (e.g., WP1130) blocks USP9X and allows targeting of polyubiquitinated $\mathrm{ALDH} 1 \mathrm{~A} 3$ to proteasome-mediated protein degradation, resulting in the loss of ALDH1A3 and cancer stem cell phenotypes.

mesenchymal markers such as CD44, C/ $\mathrm{EBP} \beta$, and TAZ, and impairs self-renewal and in vivo tumorigenicity of mesenchymal GSCs, validating the pivotal role of USP9X-ALDH1A3 axis in the maintenance of mesenchymal GSCs. Immunohistochemical analysis of 138 clinical glioblastoma specimens revealed a positive correlation between USP9X and ALDH1A3 staining, and an association of high tumor USP9X with poor survival outcomes in patients with mesenchymal glioblastoma. Collectively, the series of well-designed and executed experiments presented in this elegant work allowed the authors to conclude that USP9X is the key deubiquitinating enzyme that promotes the stabilization of ALDH1A3 in mesenchymal GSCs. Furthermore, the authors succeeded in elevating the clinical relevance of their findings by testing a small molecule that inhibits USP9X. USP9X inhibitor WP1130 (Degrasyn) recapitulated the effects observed with USP9X knockdown; it induced accumulation of poly-ubiquitinated ALDH1A3 with proteasomal inhibition, markedly reduced ALDH1A3 levels along with other mesenchymal proteins, and suppressed GSC sphere formation in vitro. Local treatment with WP1130 by 7-day continuous convection-enhanced delivery significantly inhibited the growth of mesenchymal GSCs in the brain in vivo.

\section{Inhibition of USP9X in}

\section{ALDH1A3-driven cancers}

The work by Chen et al. thus showed that ALDH1A3 is indeed the principal facilitator of the stem-like and malignant characteristics of mesenchymal GSCs, confirming prior reports. Their work is, however, unique as it for the first time identified a posttranslational molecular mechanism that regulates ALDH1A3; the deubiquitinase enzyme USP9X promotes the stability of ALDH1A3 by removing "degrons" (linkage-specific degradation signals), preventing its degradation by the proteasome (9). USP9X, also called FAM, is encoded by USP9X, located on the $\mathrm{X}$ chromosome, and a member of the ubiquitin-specific proteases (USP) family that makes up the largest subclass of DUBs and is implicated in the regulation of carcinogenesis and cancer stem cells $(10,11)$. Accumulating evidence supports oncogenic roles of USP9X, as it promotes TGF- $\beta$ / SMAD4 signaling (12) and epithelial/mesenchymal transition (13) and stabilizes antiapoptotic protein MCL1 (14) and the family of the inhibitors of apoptosis proteins (IAPS) (e.g., XIAP)(15). In glioma, USP9X promotes growth through deubiquitination and resulting stabilization of $\beta$-catenin (16) and radioresistance via, at least in part, upregulation of MCL1 (17). WP1130 was initially identified by its inhibitory action on JAK2 and found to downregulate Bcr/ $\mathrm{Abl}$ (18) and c-Myc (19). Subsequent studies attributed the pharmacological activity of WP1130 to its inhibition of the deubiquitinase activity of USP9X, USP5, USP14, and UCH37 (20). The partially selective deubiquitinase inhibition by WP1130 has been explored for cancer therapy, as WP1130 exhibits anticancer effects both as monotherapy and in combination with other modalities, such as chemotherapy, in preclinical models of a variety of cancer types, including glioblastoma $(10,11,21)$. Chen and colleagues now provide a mechanistic insight and rationale for the therapeutic inhibition of USP9X in cancers driven or sustained by ALDH1A3, represented by mesenchymal GSCs and glioblastoma.

\section{Future directions}

Despite the exciting scientific progress enabled by this publication, however, future research is necessary to address questions and challenges that remain unanswered. First, the clinical development of the USP9X inhibitor will require improvements in selectivity and drug properties. Peterson et al. developed a small molecule inhibitor of USP9X, EOAI3402143, that had better drug-like properties than WP1130 and showed antimyeloma activity (22). Second, as is the case with many other molecular targeted approaches, resistance to USP9X blockade may emerge. Indeed, USP9X knockdown leads to compensatory upregulation of USP24, a deubiquitinase closely related to USP9X, which is inhibited by EOAI3402143 (22). In the context of mesenchymal GSCs, loss of mesenchymal identity may trigger a "mesenchymal-to-proneural" switch for the GSCs to survive, and this possibility may need to be ruled out. Third, USP9Xmediated deubiquitination regulates a variety of cellular processes, including those potentially critical physiologically (23). For example, USP9X regulates DNA replication fork stability during the $\mathrm{S}$ phase (24) and the spindle assembly checkpoint during the $\mathrm{M}$ phase (25) to protect genomic stability. Thus, consequences of USP9X inhibition, even if very selective, could be diverse and may accompany unwanted or protumorigenic effects, necessitating an increased understanding of a full spectrum of USP9X functions, careful assessment of potential toxicity to normal 
tissues, and considerations for tumorselective drug delivery.

Address correspondence to: Hiroaki Wakimoto, CPZN-3800, 185 Cambridge Street, Boston, Massachusetts 02114, USA. Phone: 617.643.5987; Email: hwakimoto@ mgh.harvard.edu.

1. Omuro A, DeAngelis LM. Glioblastoma and other malignant gliomas: a clinical review. JAMA. 2013;310(17):1842-1850.

2. Lathia JD, Mack SC, Mulkearns-Hubert EE, Valentim CL, Rich JN. Cancer stem cells in glioblastoma. Genes Dev. 2015;29(12):1203-1217.

3. Verhaak RG, et al. Integrated genomic analysis identifies clinically relevant subtypes of glioblastoma characterized by abnormalities in PDGFRA, IDH1, EGFR, and NF1. Cancer Cell. 2010;17(1):98-110.

4. Mao P, et al. Mesenchymal glioma stem cells are maintained by activated glycolytic metabolism involving aldehyde dehydrogenase $1 \mathrm{~A} 3$. Proc Natl Acad Sci U S A. 2013;110(21):8644-8649.

5 . Rasper M, et al. Aldehyde dehydrogenase 1 positive glioblastoma cells show brain tumor stem cell capacity. Neuro-oncology. 2010;12(10):1024-1033.

6. Duan JJ, Cai J, Guo YF, Bian XW, Yu SC. ALDH1A3, a metabolic target for cancer diagnosis and therapy. Int JCancer. 2016;139(5):965-975.

7. Cheng $\mathrm{P}$, et al. FOXD1-ALDH1A3 signaling is a determinant for the self-renewal and tumorigenicity of mesenchymal glioma stem cells. Cancer Res. 2016;76(24):7219-7230.
8. Chen Z, et al. USP9X deubiquitinates ALDH1A3 and maintains mesenchymal identity in glioblastoma stem cells. J Clin Invest. 2019;129(5):2043-2055.

9. Kwon YT, Ciechanover A. The ubiquitin code in the ubiquitin-proteasome system and autophagy. Trends Biochem Sci. 2017;42(11):873-886.

10. Kaushal K, Antao AM, Kim KS, Ramakrishna S. Deubiquitinating enzymes in cancer stem cells: functions and targeted inhibition for cancer therapy. Drug Discov Today. 2018;23(12):1974-1982.

11. Harrigan JA, Jacq X, Martin NM, Jackson SP. Deubiquitylating enzymes and drug discovery: emerging opportunities. Nat Rev Drug Discov. 2018;17(1):57-78.

12. Dupont $S$, et al. FAM/USP9x, a deubiquitinating enzyme essential for TGF $\beta$ signaling, controls Smad4 monoubiquitination. Cell. 2009;136(1):123-135

13. Shen G, Lin Y, Yang X, Zhang J, Xu Z, Jia H. MicroRNA-26b inhibits epithelial-mesenchymal transition in hepatocellular carcinoma by targeting USP9X. BMC Cancer. 2014;14:393.

14. Schwickart M, et al. Deubiquitinase USP9X stabilizes MCL1 and promotes tumour cell survival. Nature. 2010;463(7277):103-107.

15. Karpel-Massler G, et al. Inhibition of deubiquitinases primes glioblastoma cells to apoptosis in vitro and in vivo. Oncotarget. 2016;7(11):12791-12805.

16. Yang B, et al. Deubiquitinase USP9X deubiquitinates $\beta$-catenin and promotes high grade glioma cell growth. Oncotarget. 2016;7(48):79515-79525.

17. Wolfsperger F, et al. Deubiquitylating enzyme USP9 $\mathrm{x}$ regulates radiosensitivity in glioblastoma cells by Mcl-1-dependent and -independent mechanisms. Cell Death Dis. 2016;7:e2039.

18. Bartholomeusz GA, et al. Activation of a novel $\mathrm{Bcr} / \mathrm{Abl}$ destruction pathway by WP1130 induces apoptosis of chronic myelogenous leukemia cells. Blood. 2007;109(8):3470-3478.

19. Bartholomeusz G, Talpaz M, Bornmann W, Kong LY, Donato NJ. Degrasyn activates proteasomaldependent degradation of c-Myc. Cancer Res. 2007;67(8):3912-3918.

20. Kapuria V, Peterson LF, Fang D, Bornmann WG, Talpaz M, Donato NJ. Deubiquitinase inhibition by small-molecule WP1130 triggers aggresome formation and tumor cell apoptosis. Cancer Res. 2010;70(22):9265-9276.

21. Lei H, Shan H, Wu Y. Targeting deubiquitinating enzymes in cancer stem cells. Cancer Cell Int. 2017;17:101.

22. Peterson LF, et al. Targeting deubiquitinase activity with a novel small-molecule inhibitor as therapy for B-cell malignancies. Blood. 2015;125(23):3588-3597.

23. Murtaza M, Jolly LA, Gecz J, Wood SA. La FAM fatale: USP9X in development and disease. Cell Mol Life Sci. 2015;72(11):2075-2089.

24. McGarry E, Gaboriau D, Rainey MD, Restuccia U, Bachi A, Santocanale C. The deubiquitinase USP9X maintains DNA replication fork stability and DNA damage checkpoint responses by regulating CLASPIN during S-phase. Cancer Res. 2016;76(8):2384-2393.

25. Skowyra A, Allan LA, Saurin AT, Clarke PR. USP9X limits mitotic checkpoint complex turnover to strengthen the spindle assembly checkpoint and guard against chromosomal instability. Cell Rep. 2018;23(3):852-865. 